\title{
The monotonicity of the negative stimulus during learning
}

\author{
J. A. Deutsch and G. B. Biederman \\ NEW YORK UNIVERSITY
}

\begin{abstract}
To test explanations of why reversal of a more-trained discrimination is faster, rats were trained on two discriminations simultaneously-one discrimination received nine trials per day while the other received three trials per day. The two negative cues were then paired. The usual prediction, based on the assumption of monotonicity of the acquisition of S- would be that the more trained $S-$ would be more negative than the less-trained S-. However in a group trained for nine days, 10 out of 12 rats chose the more-trained negative stimulus, showing that its negativity is not monotonic throughout acquisition.

Problem
\end{abstract}

It has often been demonstrated that when the role of the negative and positive cues is reversed in a discrimination task, learning occurs more rapidly in animals which had received more training on the original task before such a reversal (e.g., Mackintosh, 1962, 1965). On the grounds of both common sense and theory we should expect animals to take longer to reverse a habit which had been more firmly established. Indeed, the overlearning reversal effect has not been found in several recent studies (e.g., Coate \& Gardner, 1965; Erlebacher, 1963). Two main views have been advanced to explain the paradox of a smaller number of trials to reversal after longer training. The first assumes that the organism learns during prolonged training to attend to the dimension relevant to the discrimination be to learned, and that such exclusive attention to the relevant stimuli therefore facilitates reversal. Another view maintains that the negative stimulus becomes less aversive during prolonged training because the organism as training proceeds makes fewer mistakes. As the consequences of responding to the negative stimulus become less frequent, it might be expected that the negative stimulus will become less aversive. This being the case, reversal should be faster as the organism during reversal must learn to approach the previously negative cue. Attempts to show that this second interpretation is correct have been made by forcing rats to choose the negative stimulus during overlearning, so that the relative frequency of choice of this stimulus does not decrease. Such forced animals were slower to reverse than a control group which had not been made to choose the nagative stimulus during overtraining (D'Amato \& Jagoda, 1961). However, a subsequent experiment showed that this result could be attributed to the procedure of forcing in itself. Forcing of choice of the negative stimulus during overtraining produced an increase in the number of trials to reversal in a task where overlearning alone produced no decrement in the rate of learning a reversal (D'Amato \& Jagoda, 1962). Critical evidence about the relative negativity of the negative stimulus at various stages of training is therefore leacking. Yet, as D'Amato has noted, if the curve of negativity of one of the stimuli as a function of training was non-monotonic, the necessity for an attentional theory of reversal would fall away. Such a monotonic relation has in fact been assumed (Sutherland, 1964). In the present study a method was explored to test the relative strength of stimuli at various stages of training, in an attempt to provide a more secure basis for theories in this area. The method used was as follows. A group of rats was trained on two discriminations simultaneously. Trials on the two discriminations were randomly interspersed within the block of twelve daily trials. At the end of the training period the two negative stimuli were paired to see which would be chosen. If the negative stimulus from the pair which had received more training was chosen this would be evidence that the negative stimulus decreased in aversiveness as training proceeded.

\section{Method}

The Ss were 24 Long-Evans rats, approximately 3 months of age. The stimuli were four threedimensional wooden figures, $1 / 2$ in thick mounted on plywood 3 by. 3 by $1 / 2$ in. They were a large black square $2-1 / 2$ by $2-1 / 2$ in, a small black square 1 by 1 in, three horizontal stripes and three vertical stripes 3 by $1 / 2$ in each. At the center of each stimulus was a hole through which the water nozzle mounted through the wall of the chamber could be inserted, when the stimuli were in position on the wall. Thus any two stimuli could be paired in the apparatus. Ss were given five days of pretraining under $23 \mathrm{hr}$. water deprivation, food ad lib, at the two water nozzles in the divided section of the apparatus. The discriminanda were absent during pretraining. Care was taken that no $\mathrm{S}$ drank a disproportionate amount at one side of the apparatus. During training, which lasted nine days for Group 1 and 11 days for Group 2, each S was trained on two discriminations. The more-trained discrimination received nine trials per day, the less-trained discrimination was given three trials per day. Ss were randomly assigned to a group. Stimuli were completely counterbalanced across Ss with the restriction that discriminations were always horizontal vs。 vertical, and large vs. small, i.e., St, S-, more-trained and less-trained, were counterbalanced. On training days the discriminanda for a given trial and the side of 


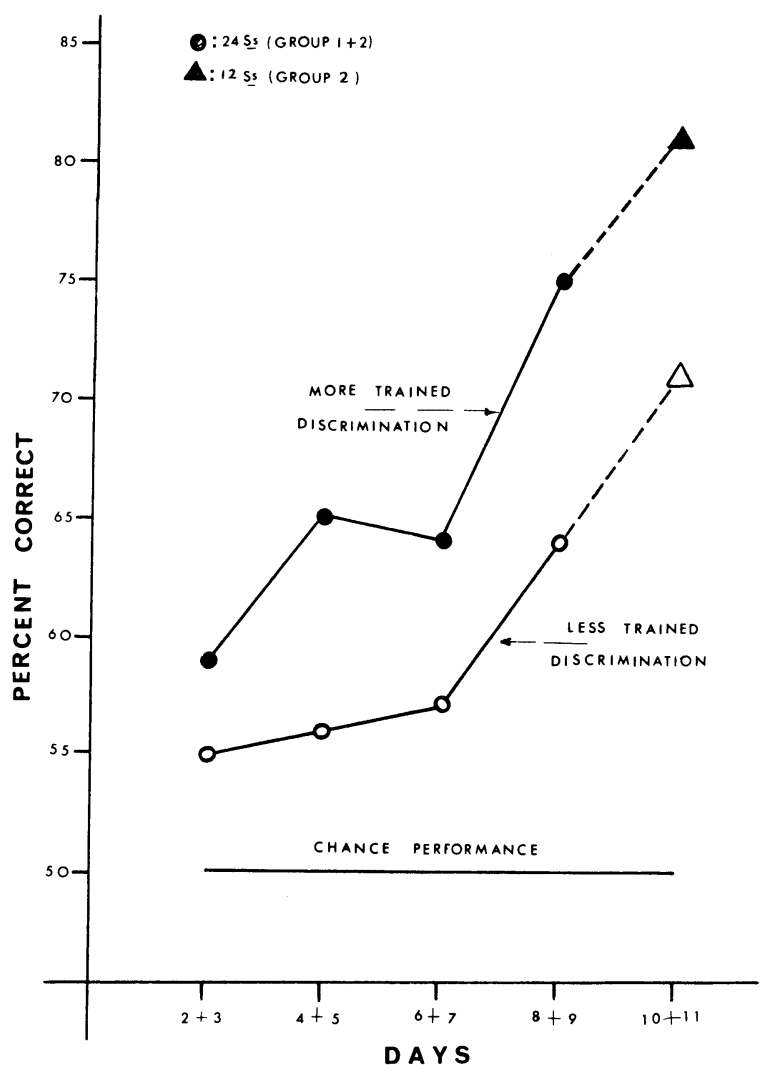

Fig. 1. Acquisition curves of more and less trained discriminations.

S+ were randomized with the restriction that one discrimination appear nine times and the other three, for a total of 12 trials per day, and that S+ not appear on one side more than three times in a row. Both $\mathrm{S}+$ and S- had water available to obviate S's detecting the presence of water through smell. Drinking was permitted for 2 sec., only at S+. A trial began when E lifted the guillotine door and was terminated when S drank at S+. $S$ was removed from $S-$ after an incorrect choice before he could drink and was replaced in the start box. $S$ could then correct an error on a given trial; there was a 15 sec. ITI. At the end of the ninth training day for Group 1 or the eleventh training day for Group 2, a thirteenth trial was presented to Sconsisting of moreand less-trained negative stimuli, and the test choice was permitted. For half the Ss, randomly selected, the more-trained (S-) stimulus was on the right.

\section{Results}

The main result shows that 10 out of 12 animals in the nine day training group chose the negative stimulus which belonged to the more-trained discrimination $(p=.02)$. At the end of training (taking the results of the last two days) the rats were choosing the correct discrimination $80 \%$ of the time in the case of the more-trained discrimination and $64 \%$ in the case of the less-trained discrimination. There were wide variations in the amount of learning which had taken place from one rat to another during training in which they had received an equal number of trials. This suggests that the decrease in relative aversiveness of a negative stimulus as training proceeds is not simply a characteristic of one stage of learning. However, it seems probable that the difference in relative aversiveness of the negative stimuli does diminish with progressive training. A second group of 12 animals (the 11 day group) was trained under identical conditions until the learning of both problems was more nearly identical ( $81 \%$ and $71 \%$ respectively). Here seven out of the 12 rats chose the more-trained negative stimulus (see Fig. 1).

Though a smaller proportion of runs was made to the negative stimulus of the more-trained pair on the last two days before the test, their absolute number was actually larger. The first group of animals had 44 errors on the more-trained stimuli and only 26 on the less-trained. The second group had 42 errors on the more-trained and 21 on the less-trained discrimination.

\section{Diseussion}

The results clearly contradict the notion that there is a monotonic increase in the aversiveness of the negative stimulus as a function of training. According to such a notion, the majority of the animals in both groups should have chosen the less-trained negative stimulus. Therefore the explanation in terms of learning to attend to a relevent dimension to account for faster reversal after overtraining may well be unnecessary. If there is no actual increase in the aversiveness of a negative stimulus with training, then faster learning of a reversal by an animal with more experience of a situation constitutes no paradox. The hypothesis that the negative stimulus becomes less aversive during training because of a lessening experience of it as learning proceeds cannot account for the present findings, since, as was stated, animals made more errors in the more-trained discrimination on the last two days. Recent failures to find the overlearning reversal effect may be due to reversing the discrimination at an adventitious level in the S- negativity acquisition curve, if it is not monotonic.

\section{References}

Coate, W. B., \& Gardner, R. A. Sources of transfer from original training to discrimination reversal. J. exp. Psychol., 1965, 70, 94-97.

D'Amato, M. R., \& Jagoda, H. Analysis of the role of overlearning in discrimination reversal. J. exp. Psychol., 1961, 61, 45-50.

D'Amato, M. R., \& Jagoda, H. Overlearning and position reversal. J. exp. Psychol., 1962, 64, 117-122.

Erlebacher, A. Reversal learning in rats as a function of percentage of reinforcement and degree of learning. J. exp. Psychol., 1963, 66, 84-90.

Mackintosh, N. J. The effects of overtraining on a reversal learning in the rat. J. comp. physiol. Psychol., 1962, 55, 555-559.

Mackintosh, N. J. Overtraining reversal and extinction in rats and chicks. J. comp. physiol. Psychol., 1965, 59, 31-36,

Sutherland, N. S. Visual discriminalion of horizontal and vertical rectangular by rats in a new discrimination training apparatus. Quart. J. exp. Psychol., 1961, 13, 117-121.

Sutherland, N. S. The learning of discrimination by animals. Endeavor, $1964,23,148-152$. 